\title{
SOLVENT-FREE MICROWAVE MICHAEL ADDITION OF ISATIN AND ANILINE SCHIFF BASE OF ISATIN TO $\alpha, \beta$-UNSATURATED ESTERS
}

\author{
GHOLAMHASSAN IMANZADEH ${ }^{a^{*}}$, TOORAN AGHAALIZADEH ${ }^{a}$, MOHAMMADREZA ZAMANLOO , \\ YAGOUB MANSOORI ${ }^{a}$
}

\author{
${ }^{a}$ Department of Chemistry, College of Science, University of Mohaghegh Ardabili 56199-11367, Ardabil, Iran
}

(Received: July 19, 2010 - Accepted: March 26, 2011)

\begin{abstract}
The rapid, simple, microwave-assisted Michael addition of isatin and aniline Schiff base of isatin to a wide variety of alkyl and aryl acrylates in the presence of, tetrabutylammonium bromide (TBAB) and 1,4-diazabicyclo[2.2.2]octane (DABCO), under solvent-free conditions, is presented. Under these conditions the addition of isatin to some of alkyl and aryl acrylates afford both related Baylis-Hillman adducts as minor products and the corresponding Michael adducts as major products. Addition of isatin to ethyl acrylate leads to produce Baylis-Hillman/Michael adduct in good yield. This system dose not work on Michael acceptors of methyl acrylate and methyl methacrylate.
\end{abstract}

Keywords: Aza-Michael addition, Microwave, Isatin, Isatin Schiff base, $\alpha, \beta$-Unsaturated Esters.

\section{INTRODUCTION}

In1841, isatin 1 (1H-indol-2,3-dione) was obtained by oxidation of indigo with nitric and chromic acids. ${ }^{1}$ Isatin and its derivatives have proved to be versatile starting material for the synthesis of natural products, heterocyclic, and non-cyclic compounds. ${ }^{2}$ Isatin and some of its derivatives are also pharmacologically relevant, for example isatin inhibits natriuretic peptide-induced hyperthermia in rats, ${ }^{3}$ and some of its semicarbazones show anticonvulsive activity. ${ }^{4}$ In nature, isatin is found in plants of the genus Isatis and in human as it is a metabolic derivative of adrenaline. ${ }^{6}$ This important compound as also been found to be a component of coal tar. ${ }^{7}$ Analogs and derivatives of isatin have found wide applications in the manufacture of dyes, pigments, flavors, pharmaceuticals. In industries, isatin and its derivatives have been also used as UV light absorbers, blowing agents, flame-proofing agents, corrosion inhibitors, dry bleaches, disinfectants, and sanitizing agents. ${ }^{8}$ Additionally, Schiff bases and Mannich basses of isatin were reported to possess antibacterial, ${ }^{9}$ antifungal, ${ }^{10}$ antiviral, ${ }^{11}$ anti-HIV,${ }^{12}$ antiprotozeal,${ }^{13}$ and antihelminthic ${ }^{14}$ activities.

Michael addition is an important and useful reaction in organic synthesis. ${ }^{15}$ In this field, recently, many attentions have been focused on the aza-Michael reaction for the construction of valuable compounds such as $\beta$-amino carbonyl compounds ${ }^{16}$ which are attractive precursors in preparation of a variety of biologically important natural products, antibiotics, $\beta$-amino alcohols, and chairal auxiliaries. ${ }^{17-22}$

In contrast to the various aza-Micheal addition methods, there are few examples of the addition of amides and their analogs, due to their low reactivity, to $\alpha, \beta$-unsaturated systems. Neutral amides have very restricted nucleophilicity but under strong basic conditions, nitrogen anions derived from them can take part in Michael-type additions to $\alpha, \beta$-unsaturated compounds. ${ }^{23}$ For instance, in the $\mathrm{NaOH}$ media the addition of $\delta$-valerolactam to ethyl acrylate produces the desired $\mathrm{N}$-substituted of this lactam in $5 \%$ yield. ${ }^{24}$ In the other media such as $\mathrm{CsF}-\mathrm{Si}(\mathrm{OR})_{4}$ this aza-Michael addition affords the corresponding $\mathrm{N}$-substituted lactam in $95 \%$ yield. ${ }^{25}$

Although, there are a few reports in literature on the aza-Michael addition of isatin to $\alpha, \beta$-unsaturated systems. ${ }^{26,27}$ However, no data are available on the addition of isatin Schiff base to these systems. In view of the emerging application of microwave (MW) chemistry in solvent-free reaction medium ${ }^{28-30}$ and in keeping with our ongoing program on the development of cleaner pathways, ${ }^{31-34}$ herein we report microwave assisted Michael addition of isatin and its aniline Schiff base (2) to $\alpha, \beta$-unsaturated esters in the presence of tetrabutylamonium bromide (TBAB) and 1,4-Diazabicyclo[2.2.2]octane $(\mathrm{DABCO})$ by using the microwave irradiation (Scheme 1).

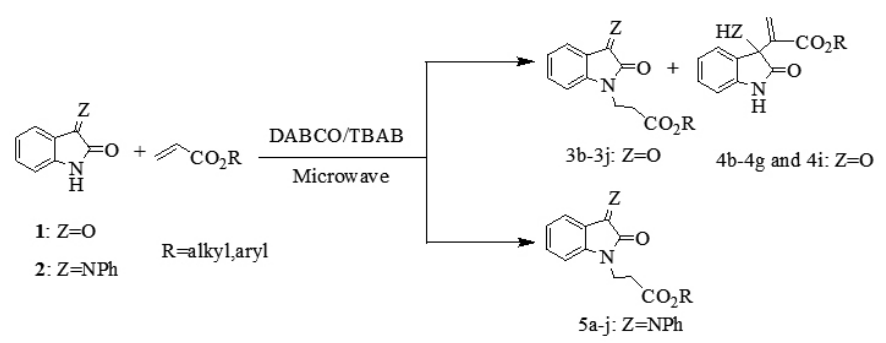

Scheme 1.

\section{RESULTS AND DISCUSSION}

Firstly, we focused on the reaction of isatin and n-butyl acrylate under MW irradiation condition. We found that reaction was completed in the presence of $\mathrm{DABCO}$ and in TBAB media at $300 \mathrm{~W}$ of microwave power. Under these conditions the aza-Michael adduct as a major product and Baylis-Hillman adduct as a minor product were obtained. Using these optimized reaction conditions, the scope and efficiency of this method was explored for a wide variety of alkyl and aryl acrylates. The results are summarized in Table 1. As Table 1 indicates, in some cases (Table 1, entries 2-7 and entry 9) the BaylisHillman adduct of isatin is formed along with Michael adduct as a side product. Surprisingly, treatment of ethyl acrylate with isatin under optimized conditions produced the Baylis-Hillman/Michael adduct in good yield (Table1, entry 1). Methyl acrylate methyl methacrylate did not undergo the Michael addition and Baylis-Hilman addition reaction (Table 1, entries 11 and entry 12). In contrast to these results, exposure of isatin to 1-phenylethyl acrylate and to 2-isopropyl5 -methylcyclohexyl acrylate under the same conditions gave the desired Michael adducts in $40 \%$ and $60 \%$ yield respectively as the sole products (Table 1 , entries 8,10 ). 
Table 1. Reaction of isatin wth different $\alpha, \beta$-unsaturated esters in the presence of $\mathrm{DABCO}$ and $\mathrm{TBAB}$ under the solvent-free microwave $(300 \mathrm{~W})$ conditions.

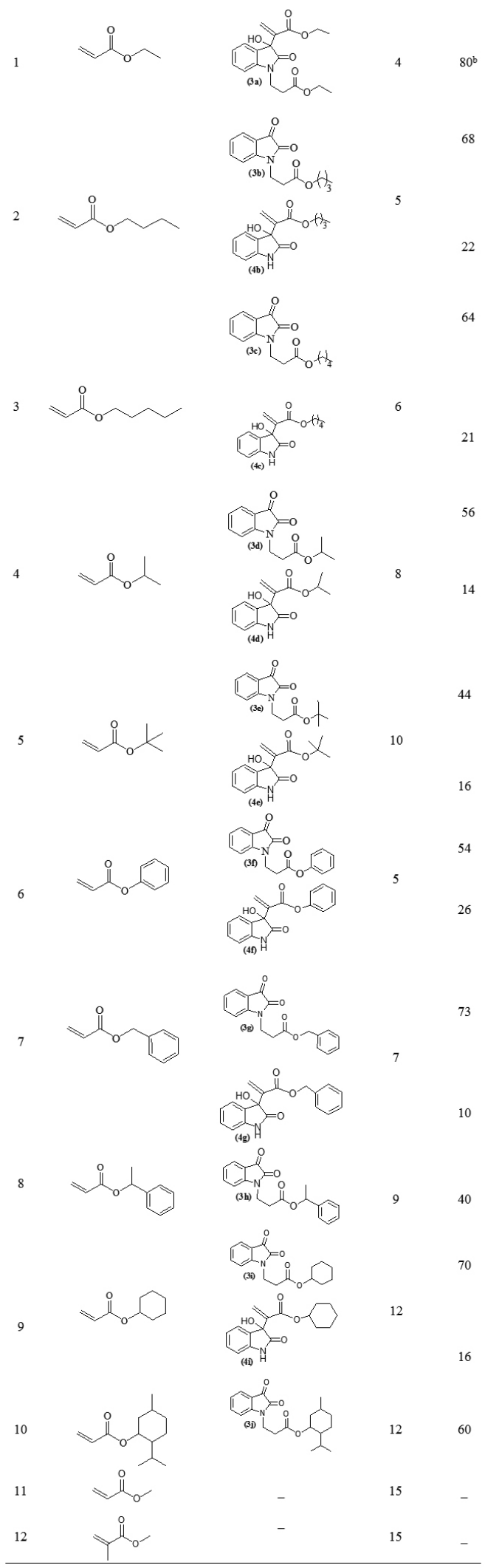

a Isolated yields. Reactions were performed using $2 \mathrm{mmol}$ ester, $1 \mathrm{mmol}$ $\mathrm{DABCO}$, and $1 \mathrm{mmol}$ TBAB. ${ }^{\text {'In }}$ this case $4 \mathrm{mmol}$ ester was used.
These results encouraged us to investigation the Michael addition of isatin Schiff base $\mathbf{2}$ to different alkyl and aryl acrylates under the same reaction conditions that the obtained results are summarized in Table 2. It is noteworthy to mention that in these reactions no Baylis-Hillman adducts are observed, and Michael adducts are produced as the sole products in good to excellent yields (Table 2). Our attempts to expedite reaction of isatin Schiff base $\mathbf{2}$ with methyl acrylate as well as with methyl methacrylate under microwave irradiation were unsuccessful.

Table 2. Aza-Micheal reaction of isatin Schiff base to $\alpha, \beta$-unsaturated esters catalyzed by DABCO and MW $(300 \mathrm{~W})$.

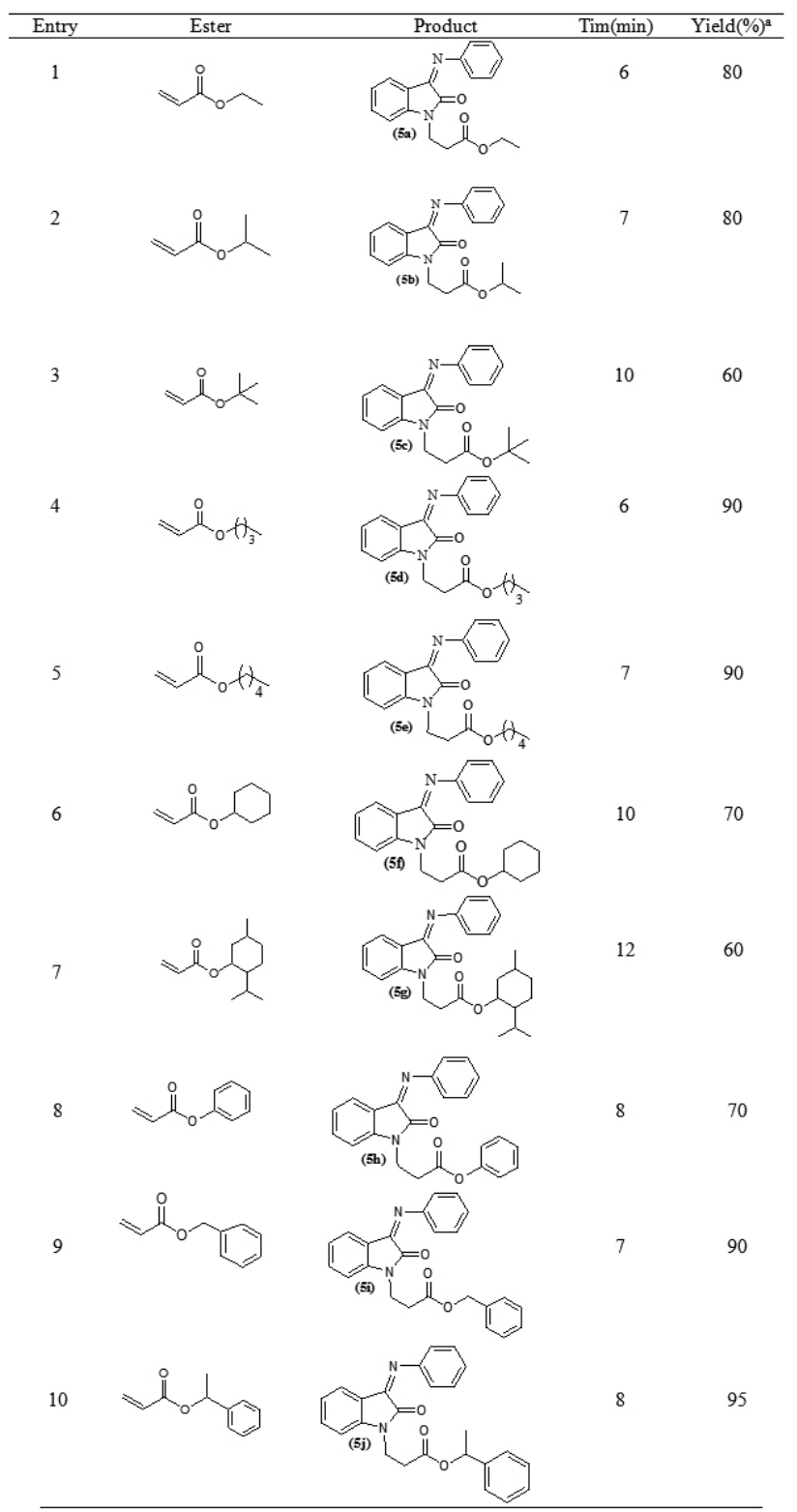

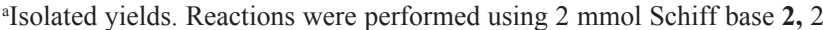
mmol ester, $1 \mathrm{mmol}$ DABCO, and $1 \mathrm{mmol}$ TBAB.

To investigation the effect of the applied base on this Michael reaction, we performed the reaction of Schiff base $\mathbf{2}$ with n-butyl acrylate in the presence of several different catalysts under the similar reaction conditions. The results are summarized in Table 3. From these results we can see that, in this reaction system, basic catalyst is necessary to make it proceed practically. Between these catalysts, DABCO, possibly for its two base functional groups, promoted the reaction much more efficiently. 
Table 3. Michael reaction of Schiff base 2 with n-butyl acrylate in the presence of different catalysts and TBAB under MW $(300 \mathrm{~W})$.

\begin{tabular}{|c|c|c|c|}
\hline Entry & Base $^{\mathrm{a}}$ & Time $(\min )$ & Yield(\%) \\
\hline 1 & None & 30 & - \\
\hline 2 & $\mathrm{DABCO}$ & 6 & 90 \\
\hline 3 & $\mathrm{NEt}_{3}$ & 15 & 50 \\
\hline 4 & $\mathrm{Na}_{2} \mathrm{CO}_{3}$ & 15 & 60 \\
\hline 5 & $\mathrm{Pyridine}^{\mathrm{a}}$ & 16 & 15 \\
\hline 6 & $\mathrm{SrCO}_{3}$ & 20 & - \\
\hline 7 & $\mathrm{NaHCO}_{3}$ & 16 & 15 \\
\hline 8 & $\mathrm{~K}_{2} \mathrm{CO}$ & 15 & 50 \\
\hline 9 & $\mathrm{MgO}$ & 20 & - \\
\hline
\end{tabular}

${ }^{a}$ With $1 \mathrm{mmol}$ base, $2 \mathrm{mmol}$ Schiff base 2, $2 \mathrm{mmol}$-butyl acrylate and 1 mmol TBAB. ${ }^{\mathrm{b}}$ Isolated yield.

According to the reaction phenomenon and results, we proposed the reaction of isatin and acrylates in the presence of DABCO may proceed as Scheme 2 and Scheme 3.
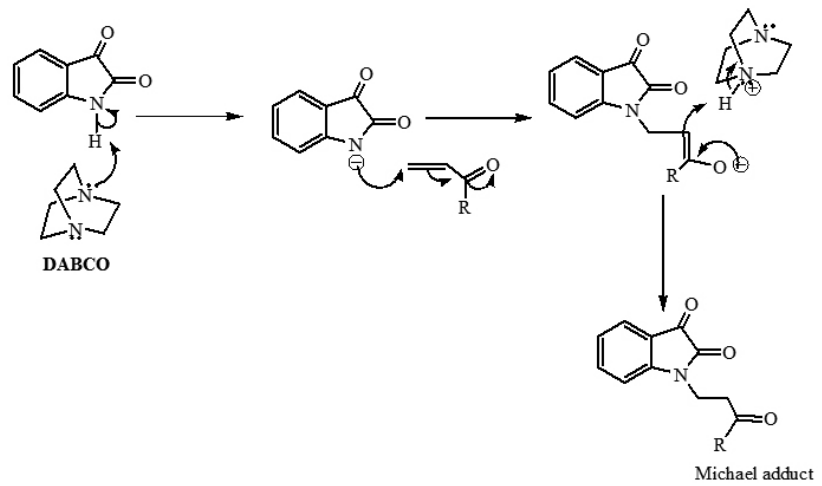

Scheme 2.

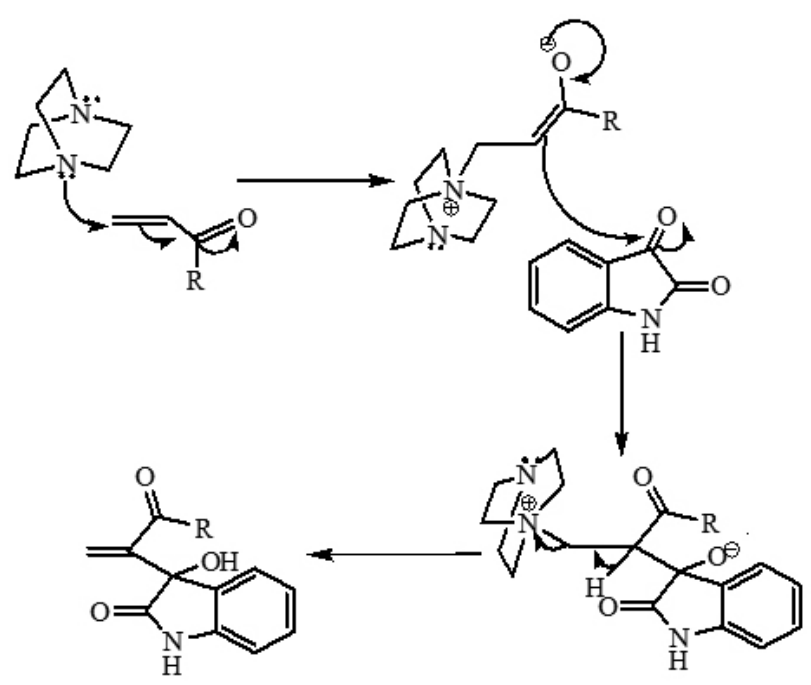

Baylis-Hilman adduct

Scheme 3.

\section{CONCLUSIONS}

In summary, we presented a rapid, simple, microwave-promoted method for Michael addition reaction of isatin and aniline Schiff base of isatin to alkyl and aryl acrylates in the presence of DABCO and TBAB. The reactions were performed at $300 \mathrm{~W}$ of microwave power under solvent-free conditions. The reaction was not successful on methyl acrylate as well as on methyl methacrylate as Michael acceptors. In some cases, isatin Michael acceptor afforded Baylis-Hilman adduct as side product. It was observed that treatment of isatin with 1-phenylethyl acrylate and with 2-isopropyl-5-methylcyclohexyl acrylate gave the desired Michael adducts as the sole products. Surprisingly, it was, also, observed that the reaction of ethyl acrylate with isatin produced the Baylis-Hillman/Michael adduct in good yield.

\section{EXPERIMENTAL}

TBAB was purchased from Fluka and DABCO, isatin, acryloylchloride and alcohols were purchased from Merck companies. All alkyl and aryl acrylates were synthesized in our laboratory according to the literature procedure and their structures were confirmed by IR and ${ }^{1} \mathrm{H}$ NMR spectra. The progress of the reactions was followed by TLC using silica gel SILIG/UV 254 plates. ${ }^{1} \mathrm{H}$ NMR (400 MHz) and ${ }^{13} \mathrm{C}$ NMR (100 MHz) spectra were recorded on Bruker 400 Spectrospin, or ${ }^{1} \mathrm{H}$ NMR $(300 \mathrm{MHz})$ and ${ }^{13} \mathrm{C}$ NMR $(75 \mathrm{MHz})$ spectra were recorded on Bruker $300 \mathrm{MHz}$, JEOL EX-90A. IR spectra were recorded on Buck-Scientific 500 instrument. Mass spectra were recorded on a Shimadzu GC-MS-QP 1000PX. Elemental analysis for C, H, and N were performed using a Heraeus CHN-O-Rapid analyzer. The melting points were determined in open capillaries with a Stuart Melting Point Apparatus and are uncorrected. MB 245 domestic microwave oven from Butan Industrial Company was used for microwave irradiation.

General Procedure for Michael addition reaction of isatin and aniline Schiff base of isatin to alkyl and aryl acrylates. To a well mixture of isatin (2 $\mathrm{mmol})$ or isatin Schiff base (2 mmol), TBAB ( $1 \mathrm{mmol})$, DABCO $(1 \mathrm{mmol})$ in a test tube was added alkyl and aryl acrylates $(2 \mathrm{mmol})$ and mixed thoroughly with a glass rod. The resulting mixture was irradiated in a microwave oven at $300 \mathrm{~W}$ for the stipulated time (Table 1 and Table 2). Then, the reaction mixture was cooled to room temperature and dissolved in chloroform $(40 \mathrm{ml})$. TBAB was recovered by the addition of water $(15 \mathrm{~mL})$, then collected and dried under vacuum. The chloroform layer was washed with water $(3 * 15 \mathrm{ml})$. The organic layer was dried over anhydrous $\mathrm{Na}_{2} \mathrm{SO}_{4}$. The solvent was removed under reduced pressure and the resulting crude material was purified on short silica-gel column with ethyl acetate:n-hexane (1:9) as the eluent.

Physical and Spectroscopic Data of Isolated Products. (3a) Red oil; IR

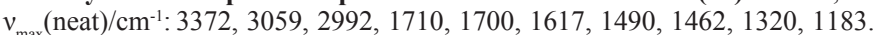
${ }^{1} \mathrm{H}$ N NMR (CDCl ${ }_{3}$, TMS, $\left.300 \mathrm{MHz}\right) \delta \mathrm{ppm}: 1.13(\mathrm{t}, 3 \mathrm{H}, J=7.2 \mathrm{~Hz}), 1.23(\mathrm{t}, 3 \mathrm{H}$, $J=7.2 \mathrm{~Hz}), 2.74(\mathrm{t}, 2 \mathrm{H}, J=7.8 \mathrm{~Hz}), 3.93-4.10(\mathrm{~m}, 7 \mathrm{H}), 6.42(\mathrm{~s}, 1 \mathrm{H}), 6.56(\mathrm{~s}$, $1 \mathrm{H}), 6.93-7.61(\mathrm{~m}, 4 \mathrm{H}) .{ }^{13} \mathrm{C} \mathrm{NMR}\left(\mathrm{CDCl}_{3}\right.$, TMS, $\left.75 \mathrm{MHz}\right) \delta \mathrm{ppm}: 13.9,14.7$, $32.2,36.2,60.9,61.1,75.9,108.8,110.4,122.9,123.8,124.0,127.5,139.3$, 143.3, 164.5, 171.3, 176.2. MS m/z: $347\left(73.00, \mathrm{M}^{+}\right), 330$ (58.70), 302 (39.00), 273 (94.10), 228 (69.50), 201 (35.00), 186 (91.90), 146 (100.00), 132 (84.50), 104 (27.00), 77 (34.00), 55 (49.80). Anal. Calcd for $\mathrm{C}_{18} \mathrm{H}_{21} \mathrm{NO}_{6}: \mathrm{C}, 62.24 ; \mathrm{H}$, 6.09 ; N, 4.03. Found: C, 62.36; H, 6.15; N, 4.12.

(3b) Red oil; IR $v_{\text {max }}\left(\right.$ neat) $/ \mathrm{cm}^{-1}: 3080,2955,2858,1722,1718,1700$, $1605,1457,1356,997,742 .{ }^{1} \mathrm{H}$ NMR $\left(\mathrm{CDCl}_{3}\right.$, TMS, $\left.300 \mathrm{MHz}\right) \delta \mathrm{ppm}: 0.92(\mathrm{t}$, $3 \mathrm{H}, J=7.5 \mathrm{~Hz}), 1.17-1.38(\mathrm{~m}, 2 \mathrm{H}), 1.44-1.61(\mathrm{~m}, 2 \mathrm{H}), 2.75(\mathrm{t}, 2 \mathrm{H}, J=6.9 \mathrm{~Hz})$, $3.93(\mathrm{t}, 2 \mathrm{H}, J=6.9 \mathrm{~Hz}), 4.03(\mathrm{t}, 2 \mathrm{H}, J=7.5 \mathrm{~Hz}), 6.92-7.62(\mathrm{~m}, 4 \mathrm{H}) \cdot{ }^{13} \mathrm{C} \mathrm{NMR}$ $\left(\mathrm{CDCl}_{3}, \mathrm{TMS}, 75 \mathrm{MHz}\right) \delta \mathrm{ppm}: 14.5,19.6,30.1,32.2,36.2,65.0,110.4,122.9$, $124.0,129.6,138.4,150.5,164.6,176.1,183.1 . \mathrm{MS} \mathrm{m} / \mathrm{z}: 275\left(11.00, \mathrm{M}^{+}\right), 228$ (19.70), 201 (21.80), $186(15.60), 146$ (30.00), 132 (36.80), 90 (17.00), 77 (22.00), 57 (42.10), 55 (44.50), 41 (100.00). Anal. Calcd for $\mathrm{C}_{15} \mathrm{H}_{17} \mathrm{NO}_{4}$ : C, 65.44; H, 6.22; N, 5.09. Found: C, 65.31; H, 6.40; N, 5.17.

(3c) Orange oil; IR $v_{\text {myx }}$ (neat) $/ \mathrm{cm}^{-1}: 3090,2930,2858,1725,1715,1700$, $1612,1455,1362,1184,973,730 .{ }^{1} \mathrm{H}$ NMR $\left(\mathrm{CDCl}_{3}, \mathrm{TMS}, 300 \mathrm{MHz}\right) \delta \mathrm{ppm}$ : $0.85(\mathrm{t}, 3 \mathrm{H}, J=7.2 \mathrm{~Hz}), 1.15-1.27(\mathrm{~m}, 4 \mathrm{H}), 1.54(\mathrm{t}, 2 \mathrm{H}, J=7.2 \mathrm{~Hz}), 2.73(\mathrm{t}, 2 \mathrm{H}$, $J=6.6 \mathrm{~Hz}), 3.88-4.05(\mathrm{~m}, 4 \mathrm{H}), 6.90-7.61(\mathrm{~m}, 4 \mathrm{H}) .{ }^{13} \mathrm{C} \mathrm{NMR}\left(\mathrm{CDCl}_{3}, \mathrm{TMS}\right.$, $75 \mathrm{MHz}) \delta$ ppm: $14.6,22.2,28.8,28.8,31.7,36.4,65.3,124.0,124.8,125.3$, $127.5,138.4,150.5,164.7,176.5,183.1 . M S \mathrm{~m} / \mathrm{z}: 289(15.00), 201$ (67.00), 145 (74.80), 132 (90.00), 119 (26.80), 90 (38.90), 77 (52.00), 55 (56.00), 43 (100.00), $41(89.50)$. Anal. Calcd for $\mathrm{C}_{16} \mathrm{H}_{10} \mathrm{NO}_{4}$ : C, 66.42; H, 6.62; N, 4.84. Found: C, 66.55; H, 6.43; N, 4.91 .

(3d) Red oil; IR $v_{\text {max }}$ (neat) $/ \mathrm{cm}^{-1}: 3085,2973,2954,1722,1719,1702$, 1624, 1459, 1459, 1347, $1182,991,736 .{ }^{1} \mathrm{H}$ NMR $\left(\mathrm{CDCl}_{3}\right.$, TMS, $\left.300 \mathrm{MHz}\right)$ $\delta$ ppm: $1.20(\mathrm{~d}, 6 \mathrm{H}, J=6.6 \mathrm{~Hz}), 2.72(\mathrm{t}, 2 \mathrm{H}, J=7.1 \mathrm{~Hz}), 4.02(\mathrm{t}, 2 \mathrm{H}, J=7.1$ $\mathrm{Hz})$, 4.81-5.00 (m, 1H), 6.93-7.63 (m, 4H). $\left.{ }^{13} \mathrm{C} \mathrm{NMR} \mathrm{(CDCl,} \mathrm{TMS,} 75 \mathrm{MHz}\right)$ $\delta$ ppm: 21.6 (two carbons), 32.5, 36.2, 68.7, 110.4, 122.9, 123.8, 125.5, 138.5, 150.4, 165.5, 176.1, 183.1. MS m/z: 261 (31.00), 219 (29.40), 201 (54.10), 174 (24.00), 146 (100.00), 132 (66.40), 43 (22.70). Anal. Calcd for $\mathrm{C}_{14} \mathrm{H}_{15} \mathrm{NO}_{4}: \mathrm{C}$, 64.36; H, 5.79; N, 5.36. Found: C, 64.24; H, 5.99; N, 5.38. 
(3e) Brown oil; IR $v_{\text {max }}$ (neat) $/ \mathrm{cm}^{-1}: 3085,2979,2925,1715,1605,1463$, $1356,1301,1174,979,742 .{ }^{1} \mathrm{H}$ NMR $\left(\mathrm{CDCl}_{3}\right.$, TMS, $\left.300 \mathrm{MHz}\right) \delta \mathrm{ppm}: 1.41$ (s, 9H), 2.65 (t, $2 \mathrm{H}, J=7.2 \mathrm{~Hz}), 3.99$ (t, $2 \mathrm{H}, J=7.2 \mathrm{~Hz}), 6.92-7.62(\mathrm{~m}, 4 \mathrm{H})$. ${ }^{13} \mathrm{C}$ NMR $\left(\mathrm{CDCl}_{3}, \mathrm{TMS}, 75 \mathrm{MHz}\right) \delta \mathrm{ppm}: 28.0$ (three carbons), 33.3, 36.3, $66.3,110.5,122.9,123.8,125.5,138.4,150.6,164.8,176.0,184.4 . \mathrm{MS} \mathrm{m} / \mathrm{z}$ : $275\left(4.00, \mathrm{M}^{+}\right), 219(18.00), 201(14.00), 146(20.90), 132$ (24.00), 90 (34.50), 77 (24.00), 57 (100.00), 43 (68.30), 41 (84.00). Anal. Calcd for $\mathrm{C}_{15} \mathrm{H}_{17} \mathrm{NO}_{4}$ : C, 65.44; H, 6.22; N, 5.09. Found: C, 65.52; H, 6.17; N, 5.04.

(3f) Orange oil; IR $v_{\text {meat }} / \mathrm{cm}^{-1}: 3065,2931,1730,1727,1698,1612$, 1496, 1472, 1362, 1313. ${ }^{1} \mathrm{H}$ NMR $\left(\mathrm{CDCl}_{3}\right.$, TMS, $\left.300 \mathrm{MHz}\right) \delta \mathrm{ppm}: 2.92(\mathrm{t}$, $2 \mathrm{H}, J=7.2 \mathrm{~Hz}), 4.11(\mathrm{t}, 2 \mathrm{H}, J=7.2 \mathrm{~Hz}), 6.91-7.67(\mathrm{~m}, 9 \mathrm{H}) .{ }^{13} \mathrm{C} \mathrm{NMR}\left(\mathrm{CDCl}_{3}\right.$, TMS, $75 \mathrm{MHz}) \delta$ ppm: 31.9, 36.0, 110.4, 121.4 (two carbons), 123.2, 124.1, 125.6, 129.4 (two carbons), 129.5, 130.5, 138.9, 150.4, 166.5, 176.1, 183.8 . MS m/z: 295 (6.00, M+), 202 (100.00), 174 (85.20), 146 (25.50), 132 (96.00), 90 (36.00), 77 (77.10), 65 (22.00), $55(44.00)$. Anal. Calcd for $\mathrm{C}_{17} \mathrm{H}_{13} \mathrm{NO}_{4}: \mathrm{C}$, 69.15; H, 4.44; N, 4.74. Found: C, 69.12; H, 4.51; N, 4.66.

(3g) Red oil; IR $v_{\text {max }}$ (neat)/ $/ \mathrm{cm}^{-1}: 3049,2949,1728,1617,1462,1345$, $1253,1187,766 .{ }^{1} \mathrm{H}$ NMR $\left(\mathrm{CDCl}_{3}, \mathrm{TMS}, 300 \mathrm{MHz}\right) \delta \mathrm{ppm}: 2.82(\mathrm{t}, 2 \mathrm{H}, J=6.9$ $\mathrm{Hz}), 4.04(\mathrm{t}, 2 \mathrm{H}, J=6.9 \mathrm{~Hz}), 5.11(\mathrm{~s}, 2 \mathrm{H}), 6.99-7.61(\mathrm{~m}, 9 \mathrm{H}) .{ }^{13} \mathrm{C} \mathrm{NMR}\left(\mathrm{CDCl}_{3}\right.$, TMS, 75 MHz) $\delta$ ppm: 32.2, 36.2, 65.0, 110.5, 123.0, 123.8, 125.5, 128.4 (two carbons), 128.6, 130.3 (two carbons), 135.3, 139.4, 150.4, 164.8, 176.2, 183.2. MS m/z: 309 (7.00, M+), 203 (30.00), 190 (14.50), 146 (20.00), 132 (27.10), 91 (100.00), 77 (23.50). Anal. Calcd for $\mathrm{C}_{18} \mathrm{H}_{15} \mathrm{NO}_{4}: \mathrm{C}, 69.89 ; \mathrm{H}, 4.89 ; \mathrm{N}, 4.53$. Found: C, 69.77; H, 4.91; N, 4.74

(3h) Brown oil; IR $v_{\max }($ neat $) / \mathrm{cm}^{-1}: 3046,2944,1726,1605,1472,1352$, $1273,1185,1008,736 .{ }^{1} \mathrm{H}$ NMR $\left(\mathrm{CDCl}_{3}, \mathrm{TMS}, 300 \mathrm{MHz}\right) \delta \mathrm{ppm}: 1.51(\mathrm{~d}, 3 \mathrm{H}$, $J=6.6 \mathrm{~Hz}), 2.79(\mathrm{t}, 2 \mathrm{H}, J=6.9 \mathrm{~Hz}), 4.02(\mathrm{t}, 2 \mathrm{H}, J=6.9 \mathrm{~Hz}), 5.87(\mathrm{q}, 1 \mathrm{H}, J=6.6$ $\mathrm{Hz}), 6.95-7.55(\mathrm{~m}, 9 \mathrm{H}) .{ }^{13} \mathrm{C}$ NMR $\left(\mathrm{CDCl}_{3}\right.$, TMS, $\left.75 \mathrm{MHz}\right) \delta$ ppm: 22.1, 32.5, $36.2,73.4,110.4,117.6,123.8,125.5,126.1$ (two carbons), 128.1, 128.6 (two carbons), 138.4, 141.1, 150.4, 160.0, 170.2, 183.1. MS m/z: $323\left(8.70, \mathrm{M}^{+}\right)$, 203 (17.00), 190 (21.00), 146 (20.10), 132 (35.00), 105 (100.00), 90 (29.80), 77 (60.50), 43 (39.50), 41 (21.80). Anal. Calcd for $\mathrm{C}_{19} \mathrm{H}_{17} \mathrm{NO}_{4}$ : C, $70.58 ; \mathrm{H}$, 5.30; N, 4.33. Found: C, 70.62; H, 5.18; N, 4.30 .

(3i) Brown oil; IR $v_{\max }$ (neat)/(cm $\mathrm{cm}^{-1}: 3040,2926,2859,1722,1698,1611$, $1472,1358,1189,978,742 .{ }^{1} \mathrm{H}$ NMR $\left(\mathrm{CDCl}_{3}\right.$, TMS, $\left.300 \mathrm{MHz}\right) \delta \mathrm{ppm}: 1.24-$ $1.79(\mathrm{~m}, 10 \mathrm{H}), 2.73(\mathrm{t}, 2 \mathrm{H}, J=6.9 \mathrm{~Hz}), 4.01(\mathrm{t}, 2 \mathrm{H}, J=6.9 \mathrm{~Hz}), 4.69(\mathrm{~m}$, $1 \mathrm{H}), 6.92-7.62(\mathrm{~m}, 4 \mathrm{H}) .{ }^{13} \mathrm{C}$ NMR $\left(\mathrm{CDCl}_{3}, \mathrm{TMS}, 75 \mathrm{MHz}\right) \delta \mathrm{ppm}: 22.1$ (two carbons), 25.2, 31.5, 32.2 (two carbons), 36.3, 73.5, 110.5, 123.1, 125.5, 130.1, 138.4, 150.6, 164.0, 176.1, 183.1. MS m/z: $301\left(33.00, \mathrm{M}^{+}\right), 219(40.00), 201$ (100.00), 175 (57.10), 145 (91.00), 132 (98.20), 104 (44.10), 90 (50.60), 77 (75.00), 55 (98.40). Anal. Calcd for $\mathrm{C}_{17} \mathrm{H}_{19} \mathrm{NO}_{4}: \mathrm{C}, 67.76 ; \mathrm{H}, 6.36 ; \mathrm{N}, 4.65$. Found: C, 67.56; H, 6.51; N, 4.82.

(3j) Brown oil; IR $v_{\max }$ (neat) $/ \mathrm{cm}^{-1}: 3050,2927,1724,1612,1454,13046$, 1202, 981, 719. ${ }^{1} \mathrm{H}$ NMR $\left(\mathrm{CDCl}^{\text {max }}\right.$, TMS, $\left.300 \mathrm{MHz}\right) \delta \mathrm{ppm}: 0.655$ (d, $3 \mathrm{H}, J=7.2$ $\mathrm{Hz}), 0.81-0.88(\mathrm{~m}, 6 \mathrm{H}), 0.92-1.91(\mathrm{~m}, 9 \mathrm{H}), 2.75(\mathrm{t}, 2 \mathrm{H}, J=6.9 \mathrm{~Hz}), 4.15(\mathrm{t}, 2 \mathrm{H}$, $J=6.9 \mathrm{~Hz}), 4.62-4.72(\mathrm{~m}, 1 \mathrm{H}), 6.89-7.63(\mathrm{~m}, 4 \mathrm{H}) .{ }^{13} \mathrm{C}-\mathrm{NMR}\left(\mathrm{CDCl}_{3}\right.$, TMS, 75 MHz) $\delta$ ppm: 16.5, 22.0 (two carbons), 22.9, 25.6, 26.3, 31.3, 32.4, 36.3, 40.3, $46.8,75.1,110.6,123.8,125.5,130.6,138.5,150.6,158.3,170.5,183.3$. MS m/z: $357\left(29.50, \mathrm{M}^{+}\right), 221(100.00), 201$ (89.50), 175 (42.00), 145 (23.00), 132 (42.80), 95 (65.80), 83 (64.00), 69 (28.00), 55 (56.50), 43 (43.90). Anal. Calcd for $\mathrm{C}_{2} \mathrm{H}_{27} \mathrm{NO}_{4}$ : C, 70.56; H, 7.61; N, 3.92. Found: C, 70.88; H, 7.45; N, 3.56.

(4b) Orange oil; IR $v_{\max }($ neat $) / \mathrm{cm}^{-1}: 3410,3085,2954,2858,1702,1680$, $1605,1450,1350 .{ }^{1} \mathrm{H}$ NMR (CDCl, TMS, $\left.300 \mathrm{MHz}\right) \delta \mathrm{ppm}: 0.80(\mathrm{t}, 3 \mathrm{H}, J$ $=6.6 \mathrm{~Hz}), 1.17-127(\mathrm{M}, 2 \mathrm{H}), 1.38-1.46(\mathrm{~m}, 2 \mathrm{H}), 3.75(\mathrm{~s}, \mathrm{OH}), 4.06(\mathrm{t}, 2 \mathrm{H}, J$ $=6.6 \mathrm{~Hz}), 6.41(1 \mathrm{H}, \mathrm{s}, \mathrm{CH}$ vinyl $), 6.55(1 \mathrm{H}, \mathrm{s}, \mathrm{CH}$ vinyl $), 6.92-7.62(\mathrm{~m}, 4 \mathrm{H})$, $7.80(\mathrm{~s}, \mathrm{NH}) .{ }^{13} \mathrm{C}$ NMR $\left(\mathrm{CDCl}_{3}\right.$, TMS, $\left.75 \mathrm{MHz}\right) \delta \mathrm{ppm}: 13.8,18.5,31.7,64.8$, 76.0, 108.8, 117.6, 123.3 124.0, 127.5, 129.6, 139.3, 143.3, 171.0, 171.3. MS $\mathrm{m} / \mathrm{z}: 275\left(10.00, \mathrm{M}^{+}\right), 228(15.00), 201(25.00), 186(18.00), 146(35.00), 117$ (10.00), 90 (20.00), 57 (45.00), 41 (100.00). Anal. Calcd for $\mathrm{C}_{15} \mathrm{H}_{17} \mathrm{NO}_{4}: \mathrm{C}$, 65.44; H, 6.22; N, 5.09. Found: C, 65.70; H, 6.15; N, 4.98.

(4c) Orange oil; IR $v_{\max }($ neat $) / \mathrm{cm}^{-1}: 3420,3950,2935,2858,1715,1702$, $1615,1455,1450,1360{ }^{1}{ }^{\max } \mathrm{HMR}$ (CDCl, TMS, $\left.300 \mathrm{MHz}\right) \delta \mathrm{ppm}: 0.83$ (t, $3 \mathrm{H}$, $J=7.2 \mathrm{~Hz}), 1.15-1.26(\mathrm{~m}, 4 \mathrm{H}), 1.45-1.52(\mathrm{~m}, 2 \mathrm{H}), 2.33(\mathrm{~s}, \mathrm{OH}), 3.88-3.94(\mathrm{~m}$, $2 \mathrm{H}), 6.42(1 \mathrm{H}, \mathrm{s}, \mathrm{CH}$ vinyl), $6.54(1 \mathrm{H}, \mathrm{s}, \mathrm{CH}$ vinyl), 6.90-7.61 (m, 4H) 7.77 (s, $\mathrm{NH}) .{ }^{13} \mathrm{C}$ NMR $\left(\mathrm{CDCl}_{3}\right.$, TMS, $\left.75 \mathrm{MHz}\right) \delta \mathrm{ppm}: 14.1,22.2,28.8,29.0,65.2$, $75.9,108.8,117.6,122.9,125.4,127.2,129.7,139.3,143.3,171.8,171.9 . \mathrm{MS}$ m/z: $289\left(15.00, \mathrm{M}^{+}\right), 201(70.00), 191$ (10.00), 145 (78.00), 119 (30.00), 77 (53.00), 55 (55.00), 43 (100.00). Anal. Calcd for $\mathrm{C}_{16} \mathrm{H}_{19} \mathrm{NO}_{4}: \mathrm{C}, 66.42 ; \mathrm{H}, 6.62$; N, 4.84. Found: C, 66.61; H, 6.45; N, 4.79 .

(4d) Red oil; IR $v_{\max }$ (neat) $/ \mathrm{cm}^{-1}: 3411,3085,2973,2954,1702,1624$, 1472, 1340. ${ }^{1} \mathrm{H}$ NMR (CDCl, TMS, $\left.300 \mathrm{MHz}\right) \delta \mathrm{ppm}: 1.03$ (d, $6 \mathrm{H}, J=6.3$ $\mathrm{Hz}), 3.62(\mathrm{~s}, \mathrm{OH}), 4.81-4.92(\mathrm{~m}, 1 \mathrm{H}), 6.35(1 \mathrm{H}, \mathrm{s}, \mathrm{CH}$ vinyl), $6.54(1 \mathrm{H}, \mathrm{s}, \mathrm{CH}$ vinyl), 6.93-7.59 (m, 4H), $7.60(\mathrm{~s}, \mathrm{NH}) .{ }^{13} \mathrm{C}$ NMR $\left(\mathrm{CDCl}_{3}\right.$, TMS, $\left.75 \mathrm{MHz}\right) \delta$ ppm: 21.4 (two carbons), 68.4, 76.1, 108.8, 117.6, 122.9, 124.1, 127.3, 129.6, 139.5, 143.4, 170.8, 170.8. MS m/z: 261 (35.00), 219 (30.00), 201 (28.00), 174 (20.00), 146 (100.00), 132 (65.00), 43 (22.00). Anal. Calcd for $\mathrm{C}_{14} \mathrm{H}_{15} \mathrm{NO}_{4}: \mathrm{C}$, 64.36; H, 5.79; N, 5.36. Found: C, 64.18; H, 5.91; N, 5.53.

(4e) Brown oil; IR $v_{\max }$ (neat) $/ \mathrm{cm}^{-1}: 3405,3075,2979,2925,1710,1604$, 1480, 1356. ${ }^{1} \mathrm{H}$ NMR $\left(\mathrm{CDCl}_{3}\right.$, TMS, $\left.300 \mathrm{MHz}\right) \delta \mathrm{ppm}: 1.281(\mathrm{~s}, 9 \mathrm{H}), 2.66$ (s, OH), $6.23(1 \mathrm{H}, \mathrm{s}, \mathrm{CH}$ vinyl), $6.63(1 \mathrm{H}, \mathrm{s}, \mathrm{CH}$ vinyl), 6.92-7.623 (m, 4H), 7.66 (s, NH). ${ }^{13} \mathrm{C}$ NMR $\left(\mathrm{CDCl}_{3}, \mathrm{TMS}, 75 \mathrm{MHz}\right) \delta$ ppm: 27.7 (three carbons), $76.1,108.8,117.6,121.5,124.1$ (two carbons), 126.8, 129.4, 140.2, 143.1, 170.5, 170.1. MS m/z: $275\left(5.00, \mathrm{M}^{+}\right), 219(10.00), 201(15.00), 174(10.00)$ 146 (20.00), 132 (25.00), 119 (12.00), 104 (10.00), 90 (35.00), 77 (25.00), 57 (100.00), 43 (58.00), 41 (84.00). Anal. Calcd for $\mathrm{C}_{15} \mathrm{H}_{17} \mathrm{NO}_{4}: \mathrm{C}, 65.44 ; \mathrm{H}, 6.22$; N, 5.09. Found: C, 65.68; H, 6.15; N, 4.87 .

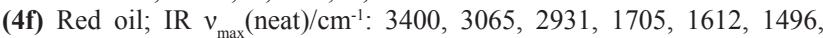
1472, 1313. ${ }^{1} \mathrm{H}$ NMR $\left(\mathrm{CDCl}_{3}\right.$, TMS, $\left.300 \mathrm{MHz}\right) \delta \mathrm{ppm}: 3.47(\mathrm{~s}, \mathrm{OH}), 6.71$ $\left(1 \mathrm{H}, \mathrm{s}, \mathrm{CH}\right.$ vinyl), $6.87\left(1 \mathrm{H}, \mathrm{s}, \mathrm{CH}\right.$ vinyl), $6.91-7.75(\mathrm{~m}, 9 \mathrm{H}), 7.97(\mathrm{~s}, \mathrm{NH}) \cdot{ }^{13} \mathrm{C}$ NMR (CDCl, TMS, $75 \mathrm{MHz}) \delta$ ppm: 75.9, 109.2, 121.4 (two carbons), 121.5, 123.2, 124.1, 126.1, 126.2, 129.3, 129.4 (two carbons), 138.5, 143.4, 150.1, 169.8, 169.9. MS m/z: $295\left(5.00, \mathrm{M}^{+}\right), 247$ (3.00), 202 (100.00), $186(5.00)$, 174 (85.00), 160 (22.00), 146 (28.00), 132 (95.00), 105 (12.00), 90 (34.00). Anal. Calcd for $\mathrm{C}_{17} \mathrm{H}_{13} \mathrm{NO}_{4}: \mathrm{C}, 69.15 ; \mathrm{H}, 4.44 ; \mathrm{N}, 4.74$. Found: $\mathrm{C}, 69.28 ; \mathrm{H}$, $4.91 ; \mathrm{N}, 4.39$

(4g) Brown oil; IR $v_{\max }($ neat $) / \mathrm{cm}^{-1}: 3436,3080,3028,2936,1709,1612$, $1465,1310 .{ }^{1} \mathrm{H}$ NMR (CDCl, TMS, $\left.300 \mathrm{MHz}\right) \delta \mathrm{ppm}: 2.69(\mathrm{~s}, \mathrm{OH}), 5.10(\mathrm{~s}$, $2 \mathrm{H}), 6.46(1 \mathrm{H}, \mathrm{s}, \mathrm{CH}$ vinyl), $6.64(1 \mathrm{H}, \mathrm{s}, \mathrm{CH}$ vinyl), 6.99-7.58 (m, 9H), $7.60(\mathrm{~s}$, $\mathrm{NH}) .{ }^{13} \mathrm{C}$ NMR $\left(\mathrm{CDCl}_{3}, \mathrm{TMS}, 75 \mathrm{MHz}\right) \delta \mathrm{ppm}: 66.7,76.6,109.0,117.6,123.0$, $123.4,124.1,128.2$ (two carbons), 128.6, 129.7, 130.3 (two carbons), 138.4, 141.1, 143.5, 168.2, 170.7. MS m/z: $309\left(8.00, \mathrm{M}^{+}\right), 203(35.00), 190(17.00)$ 175 (10.00), 161 (11.00), $146(20.00), 132$ (28.00), 119 (7.00), $105(6.00), 91$ (100.00), 77 (25.00), 65 (12.00). Anal. Calcd for $\mathrm{C}_{18} \mathrm{H}_{15} \mathrm{NO}_{4}: \mathrm{C}, 69.89 ; \mathrm{H}, 4.89$; $\mathrm{N}, 4.53$. Found: $\mathrm{C}, 69.67 ; \mathrm{H}, 4.57 ; \mathrm{N}, 4.23$.

(4i) Brown oil; IR $v_{\max }\left(\right.$ neat) $/ \mathrm{cm}^{-1}: 3470,3075,2936,1710,1611,1472$, 1358. ' ${ }^{\mathrm{H}} \mathrm{NMR}(\mathrm{CDCl}$, TMS, $300 \mathrm{MHz}) \delta \mathrm{ppm}: 1.24-2.19(\mathrm{~m}, 10 \mathrm{H}), 2.16$ (s, $\mathrm{OH}), 4.69(\mathrm{~m}, 1 \mathrm{H}), 6.37(1 \mathrm{H}, \mathrm{s}, \mathrm{CH}$ vinyl), $6.55(1 \mathrm{H}, \mathrm{s}, \mathrm{CH}$ vinyl), 6.92-7.33 $(\mathrm{m}, 4 \mathrm{H}), 8.00(\mathrm{~s}, \mathrm{NH}) .{ }^{13} \mathrm{C}$ NMR $\left(\mathrm{CDCl}_{3}, \mathrm{TMS}, 75 \mathrm{MHz}\right) \delta \mathrm{ppm:} 22.1$ (two carbons), 25.3 (two carbons), 32.2, 73.5, 76.0, 108.8, 117.6, 122.9, 123.8, 127.2, 131.5, 138.4, 143.6, 170.1, 170.4. MS m/z: $301\left(35.00, \mathrm{M}^{+}\right), 219(42.00)$, 201 (100.00), $191(20.00), 175(58.00), 163(14.00), 145$ (90.00), 132 (98.00), 119 (34.00), 104 (42.00), 90 (50.00), 83 (62.00), 77 (74.00), 67 (20.00), 55 (98.00), 41 (62.00). Anal. Calcd for $\mathrm{C}_{17} \mathrm{H}_{19} \mathrm{NO}_{4}: \mathrm{C}, 67.76 ; \mathrm{H}, 6.36 ; \mathrm{N}, 4.65$ Found: C, 67.39; H, 6.16; N, 4.45 .

(5a) Orange oil; IR $v$ (neat) $/ \mathrm{cm}^{-1}: 3059,2973,1719,1660,1599,1462$, $1348,1163,998,713 .{ }^{1} \mathrm{H}$ NMR $\left(\mathrm{CDCl}_{3}, \mathrm{TMS}, 300 \mathrm{MHz}\right) \delta \mathrm{ppm}: 1.15(\mathrm{t}, 3 \mathrm{H}$, $J=7.8 \mathrm{~Hz}), 2.78(\mathrm{t}, 2 \mathrm{H}, J=6.9 \mathrm{~Hz}), 4.55-4.07(\mathrm{~m}, 4 \mathrm{H}), 6.61-7.45(\mathrm{~m}, 9 \mathrm{H})$. ${ }^{13} \mathrm{C}$ NMR $\left(\mathrm{CDCl}_{3}\right.$, TMS, $\left.75 \mathrm{MHz}\right) \delta \mathrm{ppm}: 14.1,32.3,36.1,61.0,109.6,110.0$, 116.5, 117.7, 121.1, 122.7 (two carbons), 125.3, 126.3, 129.4 (two carbons), 134.1, 153.0, 162.1, 171.0. MS m/z: $322\left(48.00, \mathrm{M}^{+}\right), 221$ (13.00), 207 (100.00), 77 (33.00), 43 (15.00). Anal. Calcd for $\mathrm{C}_{19} \mathrm{H}_{18} \mathrm{~N}_{2} \mathrm{O}_{3}: \mathrm{C}, 70.79 ; \mathrm{H}, 5.63 ; \mathrm{N}, 8.69$. Found: C, 70.31; H, 5.61; N, 8.28.

(5b) Red oil; IR $v_{\text {max }}$ (neat) $/ \mathrm{cm}^{-1}: 3046,2968,2932,1717,1658,1611$, $1484,1361,1153,994 .{ }^{1}{ }^{\max } \mathrm{H} N \mathrm{NM}\left(\mathrm{CDCl}_{3}, \mathrm{TMS}, 300 \mathrm{MHz}\right) \delta \mathrm{ppm}: 1.23(\mathrm{~d}, 6 \mathrm{H}$, $J=7.1 \mathrm{~Hz}), 2.76(\mathrm{t}, 2 \mathrm{H}, J=7.2 \mathrm{~Hz}), 4.10(\mathrm{t}, 2 \mathrm{H}, J=7.2 \mathrm{~Hz}), 4.80-5.10(\mathrm{~m}$, $1 \mathrm{H}), 6.62-7.49(\mathrm{~m}, 9 \mathrm{H}) .{ }^{13} \mathrm{C}$ NMR $\left(\mathrm{CDCl}_{3}, \mathrm{TMS}, 75 \mathrm{MHz}\right) \delta \mathrm{ppm}: 21.7$ (two carbons), 32.6, 38.0, 76.4, 109.7, 117.7, 121.1, 122.7 (two carbons), 124.2, $125.3,126.3,129.4$ (two carbons), 133.3, 147.2, 153.1, 163.2, 173.2. MS m/z: $336\left(25.00, \mathrm{M}^{+}\right), 207(42.00), 122(17.00), 107$ (42.00), 93 (12.00), 77 (45.00), 59 (48.00), 43 (100.00). Anal. Calcd for $\mathrm{C}_{20} \mathrm{H}_{20} \mathrm{~N}_{2} \mathrm{O}_{3}: \mathrm{C}, 71.41 ; \mathrm{H}, 5.99 ; \mathrm{N}$, 8.33. Found: C, 71.18; H, 5.94; N, 8.13

(5c) Brown oil; IR $v_{\max }$ (neat) $/ \mathrm{cm}^{-1}: 3052,2949,1712,1660,1593,1161$, $1064,991,797 .{ }^{1} \mathrm{H}$ NMR $\left(\mathrm{CDCl}_{3}\right.$, TMS, $\left.400 \mathrm{MHz}\right) \delta \mathrm{ppm}: 1.41$ (s, 9H), 2.67 $(\mathrm{t}, 2 \mathrm{H}, J=7.23 \mathrm{~Hz}), 4.10(\mathrm{t}, 2 \mathrm{H}, J=7.23 \mathrm{~Hz}), 6.58-7.70(\mathrm{~m}, 9 \mathrm{H}) .{ }^{13} \mathrm{C} \mathrm{NMR}$ $\left(\mathrm{CDCl}_{3}\right.$, TMS, $\left.100 \mathrm{MHz}\right) \delta \mathrm{ppm}: 26.9$ (three carbons), 32.3, 35.1, 80.3, 108.6, 116.6, 121.6, 122.7 (two carbons), 124.2, 128.3, 133.0 (two carbons), 137.3, 145.8, 149.1, 153.1, 162.1, 169.1. MS m/z: $350\left(39.00, \mathrm{M}^{+}\right), 294(18.10), 221$ (18.10), $207(100.00), 104$ (18.00), 77 (70.90), 57 (47.50), 43 (28.50), 41 (24.00). Anal. Calcd for $\mathrm{C}_{21} \mathrm{H}_{22} \mathrm{~N}_{2} \mathrm{O}_{3}: \mathrm{C}, 71.98 ; \mathrm{H}, 6.33 ; \mathrm{N}, 7.99$. Found: $\mathrm{C}$, 71.59; H, 6.64; N, 8.17.

(5d) Red oil; IR $v_{\text {max }}($ neat $) / \mathrm{cm}^{-1}: 3052,2931,2852,1705,1599,1461,1356$, $1181,979,742 .{ }^{1} \mathrm{H}$ NMR $\left(\mathrm{CDCl}^{\max }, \mathrm{TMS}, 400 \mathrm{MHz}\right) \delta \mathrm{ppm}: 0.83(\mathrm{t}, 3 \mathrm{H}, J=7.3$ $\mathrm{Hz}), 1.21-1.31(\mathrm{~m}, 2 \mathrm{H}), 1.47-1.54(\mathrm{~m}, 2 \mathrm{H}), 2.71(\mathrm{t}, 2 \mathrm{H}, J=7.07 \mathrm{~Hz}), 3.98-4.04$ $(\mathrm{m}, 4 \mathrm{H}), 6.53-7.65(\mathrm{~m}, 9 \mathrm{H}) .{ }^{13} \mathrm{C}$ NMR $\left(\mathrm{CDCl}_{3}\right.$, TMS, $\left.100 \mathrm{MHz}\right) \delta \mathrm{ppm}: 12.3$, $17.8,29.2,31.0,34.9,63.7,108.4,116.3,121.4,122.5$ (two carbons), 124.1, 127.2, 128.1, 132.6 (two carbons), 132.9, 149.0, 153.0, 161.9, 169.8. MS m/z: 
$350\left(3.50, \mathrm{M}^{+}\right), 207$ (9.00), 93 (1100.00), 77 (7.90), 66 (36.90), 65 (19.80), 51 (7.00), 41 (13.80). Anal. Calcd for $\mathrm{C}_{21} \mathrm{H}_{22} \mathrm{~N}_{2} \mathrm{O}_{3}: \mathrm{C}, 71.98 ; \mathrm{H}, 6.33 ; \mathrm{N}, 7.99$. Found: C, 71.41; H, 6.84; N, 8.16.

(5e) Red oil; IR v (neat) $/ \mathrm{cm}^{-1}: 3052,2949,2864,1727,1666,1593,1350$, $1174,979,730{ }^{1}{ }^{1} \mathrm{H}$ NMR $\left(\mathrm{CDCl}_{3}\right.$, TMS, $\left.300 \mathrm{MHz}\right) \delta \mathrm{ppm}: 0.897$ (t, $3 \mathrm{H}, J=6.6$ $\mathrm{Hz}), 1.19-1.31$ (m, 4H), 1.50-1.62 (m, 2H), 2.796 (t, 2H, $J=7.2 \mathrm{~Hz}), 3.95-4.13$ $(\mathrm{m}, 4 \mathrm{H}), 6.61-7.46(\mathrm{~m}, 9 \mathrm{H}) .{ }^{13} \mathrm{C} \mathrm{NMR}\left(\mathrm{CDCl}_{3}\right.$, TMS, $\left.75 \mathrm{MHz}\right) \delta \mathrm{ppm}: 13.9$, $22.3,28.0,28.2,32.4,36.2,65.2,109.6,117.7,119.0,122.3$ (two carbons), 123.2, 125.3, 126.3 (two carbons), 134.1, 146.9, 150.3, 154.1, 163.2, 171.2. MS m/z: $364\left(57.00, \mathrm{M}^{+}\right), 295$ (20.00), 250 (10.00), 221 (10.00), 207 (100.00), 77 (31.00), 59 (17.00), 43 (46.00). Anal. Calcd for $\mathrm{C}_{22} \mathrm{H}_{24} \mathrm{~N}_{2} \mathrm{O}_{3}: \mathrm{C}, 72.38 ; \mathrm{H}$, $6.81 ; \mathrm{N}, 7.98$. Found: C, 72.44; H, 6.64; N, 7.69.

(5f) Brown oil; IR $v$ (neat) $/ \mathrm{cm}^{-1}: 3046,2922,2840,1721,1670,1587$, $1460,1350,1201,973,739 .{ }^{1} \mathrm{H}$ NMR $\left(\mathrm{CDCl}_{3}\right.$, TMS, $\left.400 \mathrm{MHz}\right) \delta \mathrm{ppm}: 1.19-$ $1.80(\mathrm{~m}, 10 \mathrm{H}), 2.75(\mathrm{t}, 2 \mathrm{H}, J=7.14 \mathrm{~Hz}), 4.08(\mathrm{t}, 2 \mathrm{H}, J=7.14 \mathrm{~Hz}), 4.70-4.77(\mathrm{~m}$, $1 \mathrm{H}), 6.59-7.72(\mathrm{~m}, 9 \mathrm{H}) .{ }^{13} \mathrm{C}$ NMR $\left(\mathrm{CDCl}_{3}\right.$, TMS, $\left.100 \mathrm{MHz}\right) \delta \mathrm{ppm:} 22.7$ (two carbons), 24.2, 30.5, 31.6 (two carbons), 35.2, 72.5, 108.6, 114.7, 116.7, 121.6 (two carbons), 124.3, 125.2, 128.4 (two carbons), 133.1, 145.9, 149.2, 153.1, 162.2, 169.5. MS m/z: $376\left(43.00, \mathrm{M}^{+}\right), 295$ (19.00), 221 (18.00), 207 (100.00), 104 (9.00), 77 (29.50), 55 (15.00), 41 (7.50). Anal. Calcd for $\mathrm{C}_{23} \mathrm{H}_{24} \mathrm{~N}_{2} \mathrm{O}_{3}: \mathrm{C}$, $72.73 ; \mathrm{H}, 6.58 ; \mathrm{N}, 7.62$. Found: $\mathrm{C}, 72.66 ; \mathrm{H}, 6.71 ; \mathrm{N}, 7.98$

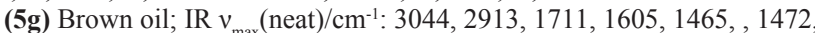
$1368,1161,973,730 .{ }^{1} \mathrm{H}$ NMR $\left(\mathrm{CDCl}_{3}\right.$, TMS, $\left.400 \mathrm{MHz}\right) \delta \mathrm{ppm}: 0.68(\mathrm{~d}, 3 \mathrm{H}$, $J=6.93 \mathrm{~Hz}), 0.82-0.88(\mathrm{~m}, 6 \mathrm{H}), 1.25-1.91(\mathrm{~m}, 9 \mathrm{H}), 2.75(\mathrm{t}, 2 \mathrm{H}, J=7.02 \mathrm{~Hz})$, $4.04(\mathrm{t}, 2 \mathrm{H}, J=7.02 \mathrm{~Hz}), 4.64-4.72(\mathrm{~m}, 1 \mathrm{H}), 6.60-7.61(\mathrm{~m}, 9 \mathrm{H}) .{ }^{13} \mathrm{C} \mathrm{NMR}$ $(\mathrm{CDCl}, \mathrm{TMS}, 100 \mathrm{MHz}) \delta \mathrm{ppm}: 15.2,20.9,22.4$ (two carbons), 25.3, 28.7, $30.4,31.4,31.6,36.8,45.8,74.2,108.8,116.7,121.7,122.8$ (two carbons), 123.7, 124.5, 125.3, 128.4 (two carbons), 133.1, 137.4, 149.6, 157.3, 169.5. MS m/z: 432 (19.50, M+), 295 28.10), 221 (35.00), 207 (100.00), 194 (19.50), 132 (18.00), 77 (66.50), 55 (66.50), 43 (40.50), 41 (42.50). Anal. Calcd for $\mathrm{C}_{27} \mathrm{H}_{32} \mathrm{~N}_{2} \mathrm{O}_{3}$ : C, 74.97; H, 7.46; N, 6.47. Found: C, 74.52; H, 7.19; N, 6.81.

(5h) Orange oil; IR $v_{\max }$ (neat) $/ \mathrm{cm}^{-1}: 3044,2913,1730,1726,1654,1599$, $1532,1472,1356,1175,1022,764 .{ }^{1} \mathrm{H} \mathrm{NMR}^{\max }\left(\mathrm{CDCl}_{3}, \mathrm{TMS}, 400 \mathrm{MHz}\right) \delta \mathrm{ppm}$ : $3.06(\mathrm{t}, 2 \mathrm{H}, J=6.98 \mathrm{~Hz}), 4.21(\mathrm{t}, 2 \mathrm{H}, J=6.98 \mathrm{~Hz}), 6.99-7.60(\mathrm{~m}, 14 \mathrm{H}) \cdot{ }^{13} \mathrm{C}$ NMR (CDCl, TMS, $100 \mathrm{MHz}) \delta$ ppm: 31.4, 35.04, 108.6, 114.3, 116.7, 119.1, 120.3 (two carbons), 121.8 (two carbons), 124.4, 125.1, 127.7, 128.4 (two carbons), 128.5 (two carbons), 133.2, 136.8, 145.7, 153.0, 162.4, 168.7. MS m/z: $370\left(27.00, \mathrm{M}^{+}\right), 277$ (100.00), 249 (28.00), 223 (55.00), 207 (75.00), 94 (22.70), 77 (65.00), 51 (21.00). Anal. Calcd for $\mathrm{C}_{23} \mathrm{H}_{18} \mathrm{~N}_{2} \mathrm{O}_{3}: \mathrm{C}, 74.58 ; \mathrm{H}, 4.90$; N, 7.56. Found: C, 74.26; H, 4.93; N, 7.90.

(5i) Red oil; IR $v_{\text {max }}($ neat $) / \mathrm{cm}^{-1}: 3046,3011,2949,1721,1654,1605$, $1520,1453,1368,1188,1009,748 .{ }^{1} \mathrm{H} \mathrm{NMR}^{\text {max }}\left(\mathrm{CDCl}_{3}, \mathrm{TMS}, 400 \mathrm{MHz}\right) \delta \mathrm{ppm}$ : $2.83(\mathrm{t}, 2 \mathrm{H}, J=7.02 \mathrm{~Hz}), 4.10(\mathrm{t}, 2 \mathrm{H}, J=7.02 \mathrm{~Hz}), 5.10(\mathrm{~s}, 2 \mathrm{H}), 6.60-7.72$ $(\mathrm{m}, 14 \mathrm{H}) .{ }^{13} \mathrm{C}$ NMR $\left(\mathrm{CDCl}_{3}\right.$, TMS, $\left.100 \mathrm{MHz}\right) \delta \mathrm{ppm}: 31.2,35.0,65.7,107.5$, 114.6, 116.5, 121.5, 122.6 (two carbons), 124.2, 125.1, 127.4 (two carbons), 128.3 (two carbons), 132.7 (two carbons), 132.9, 134.2, 145.7, 149.1, 152.9, 162.1, 169.7. MS m/z: 384 (45.00, $\left.\mathrm{M}^{+}\right)$, 221(19.50), 207 (100.00), 91 (51.00), 77 (51.90), 65 (12.50), 51 (14.00), 43 (5.50). Anal. Calcd for $\mathrm{C}_{24} \mathrm{H}_{20} \mathrm{~N}_{2} \mathrm{O}_{3}: \mathrm{C}$, 74.98; H, 5.24; N, 7.29. Found: C, 75.21; H, 5.54; N, 7.72.

(5j) Brown oil; IR $v_{\max }$ (neat) $/ \mathrm{cm}^{-1}: 3065,2967,2925,1717,1698,1658$, $1605,1490,1472,1358,1155,991,675 .{ }^{1} \mathrm{H}$ NMR (CDCl, TMS, $\left.300 \mathrm{MHz}\right) \delta$ ppm: 1.52 (d, $3 \mathrm{H}, J=6.6 \mathrm{~Hz}), 2.81(\mathrm{t}, 2 \mathrm{H}, J=7.2 \mathrm{~Hz}), 4.08(\mathrm{t}, 2 \mathrm{H}, J=7.2 \mathrm{~Hz})$, $5.57(\mathrm{q}, 1 \mathrm{H}, J=6.6 \mathrm{~Hz}), 6.59-7.45(\mathrm{~m}, 14 \mathrm{H}) .{ }^{13} \mathrm{C} \mathrm{NMR}\left(\mathrm{CDCl}_{3}, \mathrm{TMS}, 75 \mathrm{MHz}\right)$ $\delta$ ppm: 22.1, 32.6, 36.1, 73.2, 109.6, 117.7, 119.0, 122.2 (two carbons), 123.3, $125.3,126.1,126.3$ (two carbons), 128.0 (two carbons), 129.4 (two carbons), 133.8, 141.2, 146.9, 150.2, 154.1, 163.3, 170.3. MS m/z: $398\left(100.00, \mathrm{M}^{+}\right)$, 221 (21.00), 207 (91.00), 105 (30.00), 77 (28.00), 51 (8.00). Anal. Calcd for $\mathrm{C}_{25} \mathrm{H}_{22} \mathrm{~N}_{2} \mathrm{O}_{3}: \mathrm{C}, 75.36 ; \mathrm{H}, 5.57 ; \mathrm{N}, 7.03$. Found: $\mathrm{C}, 75.86 ; \mathrm{H}, 5.47 ; \mathrm{N}, 6.86$.

\section{ACKNOWLEDGEMENTS}

The authors thankful to, University of Mohaghegh Ardabili and Tabriz University Councils for the partial support to the work.

\section{REFERENCES}

[1] W. C. Sumpter, Chem. Rew. 34, 407, (1945).

[2] J. F. M. Da Sila, S. J. Garden, A. C. Pinto, J. Braz. Chem. Soc. 12, 273, (2001).

[3] I. Pataki, A. Adamik, G. Telegdy, Peptides 21, 373 (2000).

[4] S. N. Pendeya, A. S. Raja, J. P. Stables, J. Pharm. Pharmaceut. Sci. 5, 266, (2002).

[5] Y. Guo, F. Chen, Chem. Abstr. 17, 8, (1986).

[6] N. Ischia, A. Palumbo, G. Prota, Tetrahedron 44, 6441, (1988).

[7] Y. Yan, F. Wang, G. Li, W. Mao, Chem. Abstr. 18, 192, (1992).

[8] G. M. Coppola, Synthesis 505, (1980).

[9] S. N. Pandeya, D. Sriram, Acta Pharm. Turc. 40, 33, (1998).

[10] S. N. Pandeya, D. Sriram, G. Nath, E. De-Clercq, Indian J. Pharm. Sci. 61, 358, (1999).

[11] R. S. Varma, W. L. Nobles, J. Med. Chem. 10, 972, (1967).

[12] S. N. Pandeya, P. Yogeeswari, D. Sriram, E. De-Clercq, C. Pannecouque, M. Wivrouw, Chemotherapy 45, 192, (1999).

[13] S. A. Imam, R. S. Varma, Experientia 31, 1287, (1975).

[14] S. E. Sarciron, P. Audin, I. Delebre, C. Gabrion, A. F. Petvay, J. Paris, J. Pharm. Sci. 82, 605, (1993).

[15] P. Perlmutter, Conjugate Addition Reactions in Organic Synthesis, Pergamon, Oxford, 1992.

[16] G. Bartoli, C. Cimarelli, E. Marcantoni, G. Palmieri, M. Petrini, J. Org. Chem. 59, 5328, (1994).

[17] M. Tramontini, Synthesis 703, (1973).

[18] W. F. Wang, T. Izawa, S. Kobayashi, M. Ohno, J. Am. Chem. Soc. 104, $6465,(1982)$

[19] Y. Hayashi, J. J. Rode, E. J. Corey, J. Am. Chem. Soc. 118, 5502, (1996).

[20] E. Juariti, Enantioselective Synthesis of $\beta$-Amino Acids, Willey-VCH, New York, 1997.

[21] G. I. Gorge, The Organic Chemistry of $\beta$-Lactoms, Willey-VCH, New York, 1993.

[22] S. Kobayashi, H. Ishitani, Chem. Rev. 99, 1069, (1999).

[23] R. R. Mariclla, R. Jonauskas, J. Org. Chem. 23, 923, (1958).

[24] M. Maggini, M. Prato, M. Ranelli, G. Scorrano, Tetrahedron Lett. 33, $6537,(1992)$

[25] R. J. P. Corriu, R. Oerz, Tetrahedron Lett. 26, 1311, (1985).

[26] T. Ohnuma, H. Kasuya, Y. Kimura, Y. Ban, Heterocycles 17, 377, (1982).

[27] J. Frederick, D. Carlo, H.G. Lindwall, J. Am. Chem. Soc. 67, 199, (1945).

[28] J. Zou, F. Li, F.G. Tao, Chinese Chem. Lett. 20, 17, (2009).

[29] L. Perreux, A. Loupy, Tetrahedron 57, 9199, (2001).

[30] M. Chaouchi, A. Loupy, S. Marque, A. Petit, Eur. J. Org. Chem. 7, 1278, (2002).

[31] M. Ghiaci, G. H. Imanzadeh, Synth Commun. 28, 2275, (1998).

[32] G. H. Imanzadeh, A. R. Hajipour, S. E. Mallakpour, Synth. Commun. 33, 735, (2003) [33] G. H. Imanzadeh, M. R. Zamanluo, H. Eskandari, K. Shayesteh, J. Chem. Res. 151, (2006).

[34] G. H. Imanzadeh, A. Khalafinezhad, A. Zare, A. Hasaninejad, A. Mosavi Zare, A. Parhami, J. Iran. Chem. Soc. 4, 467, (2007). 